\title{
Origin of Urinary Nonconjugated 19-Nor-Deoxycorticosterone and Metabolism of Infused Radiolabeled 19-Nor-Deoxycorticosterone in Men and Women
}

\author{
M. Linette Casey, Alireza Guerami, Leon Milewich, Celso E. Gomez-Sanchez, and Paul C. MacDonald \\ The Cecil H. and Ida Green Center for Reproductive Biology Sciences and Departments of Biochemistry and Obstetrics-Gynecology, \\ The University of Texas Southwestern Medical School, Dallas, Texas 75235; Department of Medicine, \\ The University of South Florida College of Medicine, Tampa, Florida 33612
}

\begin{abstract}
It is known that 19-nor-deoxycorticosterone (19-nor-DOC) is a potent mineralocorticosteroid that is present in urine of rats and humans in a free, i.e., nonconjugated, form. In some forms of hypertension in rats, the levels of free 19-nor-DOC in urine are increased compared with those in urine of normotensive animals. Yet, despite the potential importance of this mineralocorticosteroid in the pathogenesis of certain forms of hypertension, little is known of its site of origin or metabolism. In the present investigation, we evaluated the metabolism of intravenously infused $\left[{ }^{3} \mathrm{H}\right] 19$-nor-DOC and the possibility that 19-nor-DOC was formed from plasma DOC. We found that the metabolism of $\left[{ }^{3} \mathrm{H}\right] 19-$-nor-DOC infused intravenously in men and women was similar to that of DOC with important exceptions. The majority of the radiolabeled urinary metabolites of intravenously infused $\left[{ }^{3} \mathrm{H}\right] 19-$-nor-DOC were excreted in urine as glucuronosides. Little radioactivity, infused as $\left[{ }^{3} \mathrm{H}\right] 19$ nor-DOC, was recovered in urine as nonconjugated or sulfoconjugated steroids. There was no free radiolabeled 19-norDOC in urine after the simultaneous infusion of $\left[{ }^{3} \mathrm{H}\right] 19-$-norDOC and $\left[{ }^{14} \mathrm{C}\right] D O C$. A major metabolite of $\left[{ }^{3} \mathrm{H}\right] 19-$-nor-DOC in urine was 19-nor-DOC-21-glucuronoside, whereas little or no intravenously infused radiolabeled DOC was excreted as radiolabeled DOC-glucuronoside. We also found that intravenously infused $\left[{ }^{14} \mathrm{C}\right] \mathrm{DOC}$ was not converted to urinary $\left[{ }^{14} \mathrm{C}\right] 19-$ nor-DOC (glucuronoside) and that other tritium-labeled metabolites of infused $\left[{ }^{3} \mathrm{H}\right] 19-$-nor-DOC contained no carbon-14. The production rate of 19-nor-DOC, computed from the specific activity of urinary 19-nor-DOC (glucuronoside), in one normal man was $16 \mu \mathrm{g} / \mathrm{d}$ and in the two women of this study, it was $10 \mu \mathrm{g} / \mathrm{d}$. These findings are supportive of the proposition that free urinary 19-nor-DOC is not formed from plasma DOC; it may be formed in kidney from a precursor other than DOC or it may be formed nonenzymatically in kidney or urine from a precursor such as 19-oic-DOC.
\end{abstract}

\section{Introduction}

Recently, there has been considerable interest in the potential role of 19-nor-deoxycorticosterone (19-nor-DOC) ${ }^{1}$ as a mineralocorticosteroid in physiologic and possibly pathophysiologic

Address correspondence to Dr. Casey, The Cecil H. and Ida Green Center for Reproductive Biology Sciences, The University of Texas, Southwestern Medical School, 5323 Harry Hines Blvd., Dallas, TX 75235.

Received for publication 5 July 1984 and in revised form 27 December 1984.

J. Clin. Invest.

(C) The American Society for Clinical Investigation, Inc.

0021-9738/85/04/1335/04\$\$1.00

Volume 75, April 1985, 1335-1338 processes. It is known that 19 -nor-DOC is a potent mineralocorticosteroid (1-6) that is present in nonconjugated form in urine of rats undergoing adrenal regeneration (5) and in rats with spontaneous (7) hypertension as well as in urine of normotensive animals. The levels of 19-nor-DOC in urine of these hypertensive rats appear to be greater than those in normotensive rats. From these findings, the possibility was considered that 19-nor-DOC may be instrumental in the pathogenesis of certain forms of hypertension. Recently, it also was demonstrated that free 19-nor-DOC is present in human urine (8) and that the levels of this nonconjugated steroid in urine increase after ACTH treatment (8-10). In spite of these provocative findings, however, the tissue site(s) of biosynthesis of 19-nor-DOC is unknown and the metabolic fate of this mineralocorticosteroid is not defined. For example, it was not possible to demonstrate the biosynthesis of 19-nor-DOC in rat adrenal tissue in vitro $(11,12)$.

Recently, we found that in the human, DOC is formed in extraadrenal sites from plasma progesterone (13-15). Some extraadrenal tissues of DOC formation, e.g., kidney and aorta, are believed to be target tissues of DOC action (16-19). It was of interest, therefore, to determine whether 19-nor-DOC is formed from circulating DOC and if this were the source of nonconjugated 19-nor-DOC that is present in human urine. For these reasons, the present study was undertaken to evaluate the metabolism of intravenously infused radiolabeled 19-norDOC and DOC in men and women.

\section{Methods}

Radiolabeled steroid preparation, purification, and administration. $\left[1,2(N)-{ }^{3} \mathrm{H}\right] 19$-nor-DOC $(50 \mathrm{Ci} / \mathrm{mmol})$ was synthesized as described (12) and purified by high performance liquid chromatography (HPLC) and by gradient-elution column chromatography on celite-ethylene glycol (13). $\left[{ }^{14} \mathrm{C}\right] \mathrm{DOC}(50 \mathrm{mCi} / \mathrm{mmol})$, purchased from New England Nuclear (Boston, MA), was purified as described (13). A mixture of a tracer dose that consisted of $\left[{ }^{14} \mathrm{C}\right] \mathrm{DOC}(\sim 18 \mu \mathrm{Ci})$ and $\left[{ }^{3} \mathrm{H}\right] 19-$-nor$\mathrm{DOC}(\sim 7 \mu \mathrm{Ci})$ in $4.5 \mathrm{ml} \mathrm{NaCl}(0.15 \mathrm{M})$ and $0.5 \mathrm{ml}$ ethanol was infused intravenously into two healthy, ambulatory men, ages 47 and 52 , and into two presumably ovulatory women, ages 33 and 35 ; thereafter, urine was collected from each subject for $72 \mathrm{~h}$. Nonradiolabeled 19-nor-DOC was prepared as described (5) and the authenticity of this compound was validated by use of gas chromatography-mass spectrometry.

Urine processing and metabolite purification. Free, i.e., nonconjugated, 19-nor-DOC in the urine of each test subject was quantified by radioimmunoassay as described (20). To evaluate the metabolism of the injected radiolabeled steroids, one-half of the urine collected was extracted with ethyl acetate to remove nonconjugated steroids. The other one-half of the urine was mixed with acetic acid to adjust the $\mathrm{pH}$ to 5.0. Thereafter, the acidified urine was incubated with $\beta$ -

1. Abbreviations used in this paper: DOC, deoxycorticosterone; HPLC, high performance liquid chromatography; TLC, thin-layer chromatography. 
glucuronidase for $48 \mathrm{~h}$ at $37^{\circ} \mathrm{C}$ to effect hydrolysis of steroid glucuronosides. The $\beta$-glucuronidase-treated urine was extracted with ethyl acetate. The aqueous residues were combined and treated by solvolysis (21) to effect hydrolysis of steroid sulfates. Aliquots of the residues obtained from each urine extract were assayed for radioactivity.

Radiolabeled steroids in these residues were isolated by successive chromatographic procedures that included, initially, gradient-elution column chromatography on celite-ethylene glycol (13). The fractions of this chromatogram that corresponded in mobility to $\left[{ }^{3} \mathrm{H}\right] 19$-norDOC were combined and chromatographed by liquid-liquid partition column chromatography on celite with the solvent system isooctane/ $t$-butanol/methanol/water (10:4:3:3, by volume). After this chromatographic procedure, the isolated $\left[{ }^{3} \mathrm{H}\right] 19$-nor-DOC did not contain carbon-14. The $\left[{ }^{3} \mathrm{H}\right] 19$-nor-DOC was purified further by thin-layer chromatography (TLC) on silica gel $\mathrm{GF}_{254}$ plates $(0.25 \mathrm{~mm}$; Analtech Inc., Newark, DE) by use of two different solvent systems, i.e., ethyl acetate/isooctane $(7: 3, \mathrm{vol} / \mathrm{vol})$ and benzene/ethanol $(9: 7, \mathrm{vol} / \mathrm{vol})$; thereafter, the steroid was purified by HPLC by use of a $\mathrm{C}_{18}-\mu$ Bondapak column (Waters Associates, Milford, MA) with the solvent system methanol/water $(7: 3, \mathrm{vol} / \mathrm{vol})$ in the isocratic mode (13). Subsequently, the isolated $\left[{ }^{3} \mathrm{H}\right] 19-$-nor-DOC was purified by HPLC by use of a normal phase $\mu$ Porasil column (Waters Associates) with the eluent hexane/ isopropanol $(9: 1, \mathrm{vol} / \mathrm{vol})$. The $\left[{ }^{3} \mathrm{H}\right] 19$-nor-DOC was acetylated by treatment with a mixture of pyridine and acetic anhydride $(1: 1, \mathrm{vol} /$ vol) at $37^{\circ} \mathrm{C}$ for $2 \mathrm{~h}$. Thereafter, the 19-nor-DOC-acetate was chromatographed by TLC with ethyl acetate/isooctane $(1: 1, \mathrm{vol} / \mathrm{vol})$ as the developing solvent. The specific activity of urinary $\left[{ }^{3} \mathrm{H}\right] 19$-nor-DOC (glucuronoside) was determined as described (22).

\section{Results}

Little of the radioactivity administered as $\left[{ }^{3} \mathrm{H}\right] 19-$-nor-DOC or $\left[{ }^{14} \mathrm{C}\right] \mathrm{DOC}$ was extracted from urine as "free" steroids; indeed, $<1 \%$ of tritium from injected $\left[{ }^{3} \mathrm{H}\right] 19$-nor-DOC and $<0.5 \%$ of carbon-14 from injected $\left[{ }^{14} \mathrm{C}\right] \mathrm{DOC}$ were recovered in the "free" steroid extracts. This was true despite the fact that there was immunoreactive, free, nonradiolabeled 19-nor-DOC, as determined by radioimmunoassay, in the urine of each test subject. In these persons, free 19-nor-DOC levels were 11-57 $\mathrm{ng} / 24 \mathrm{~h}$ urine. There was no free radiolabeled 19-nor-DOC or DOC in the urine of any test subject. There also was no $\left[{ }^{3} \mathrm{H}\right] 19-$-nor-DOC in extracts prepared after acid solvolysis. On the other hand, $>60 \%$ of the $\left[{ }^{3} \mathrm{H}\right] 19-$-nor-DOC and $>40 \%$ of the $\left[{ }^{14} \mathrm{C}\right] \mathrm{DOC}$ administered were recovered by ethyl acetate extraction after $\beta$-glucuronidase treatment of the urine, a finding that is indicative that most of the metabolites of 19nor-DOC and DOC are excreted into urine as glucuronosides. By gradient-elution column chromatography, we isolated five major peaks of carbon-14 radioactivity and six peaks that contained tritium radioactivity. In the case of the carbon-14 peaks, there were corresponding peaks of tritium that were slightly more polar than the carbon-14 counterpart (Fig. 1). We find, as do others $(5,12,23)$, that in most chromatographic systems, 19-nor-DOC is slightly more polar than DOC.

Radiolabeled 19-nor-DOC was one of the major tritiumlabeled metabolites isolated after $\beta$-glucuronidase treatment of urine. This steroid was purified to radiochemical homogeneity (Fig. 2) and, thereafter, the specific activity of this urinary metabolite was determined by assay of radioactivity and quantification of mass by ultraviolet absorption. Carbon-14-labeled DOC was not present in urine after $\beta$-glucuronidase treatment. Moreover, there was no carbon- 14 radioactivity in purified urinary 19-nor-DOC recovered after the intravenous administration of $\left[{ }^{3} \mathrm{H}\right] 19-$-nor-DOC and $\left[{ }^{14} \mathrm{C}\right] \mathrm{DOC}$. A second radiolabeled metabolite of $\left[{ }^{3} \mathrm{H}\right] 19-$ nor-DOC, which is presumed to be 19-nor-5 $\beta$-pregnane-3 $\alpha, 21$-diol-20-one, was purified in a similar manner, as was a third tritium-labeled metabolite, which is

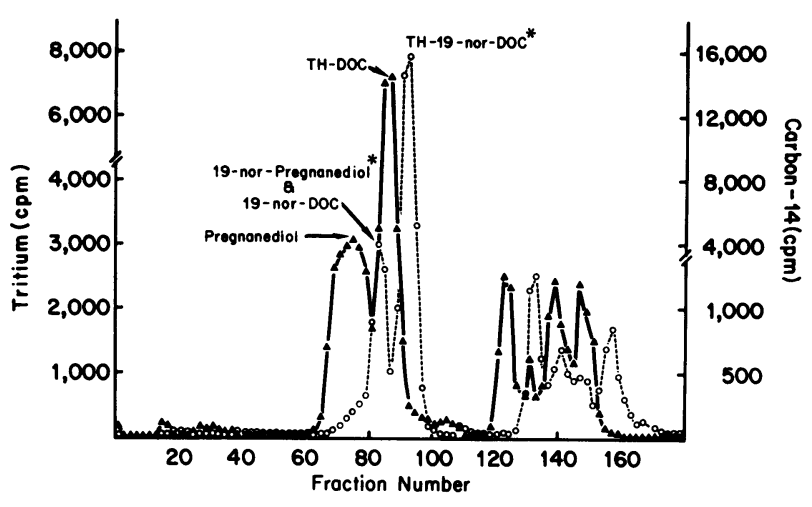

Figure 1. Chromatogram, by gradient-elution column chromatography on celite-ethylene glycol, of radiolabeled urinary steroids obtained after $\beta$-glucuronidase treatment of urine of a person that was given $\left[{ }^{3} \mathrm{H}\right] 19-$-nor-DOC and $\left[{ }^{14} \mathrm{C}\right] \mathrm{DOC}$ intravenously. Five major peaks that contained carbon- 14 and six peaks that contained tritium were identified (Figs. 1 and 2); for each peak of carbon-14 radioactivity, there is a corresponding tritium peak that is slightly more polar than its carbon- 14 counterpart. The additional peak of tritium, i.e., for which there was no correspondent peak of carbon-14, was proven to be $\left[{ }^{3} \mathrm{H}\right] 19-$ nor-DOC, indicating that little or no DOC is excreted as a glucuronoside (see text). The asterisks are indicative of tentative identification by isopolarity in multiple chromatographic systems. --- O---, tritium; - $\triangle-$, carbon-14.

presumed to be 19-nor-5 $\beta$-pregnane- $3 \alpha, 20 \alpha$-diol; neither of these purified tritium-labeled metabolites of $\left[{ }^{3} \mathrm{H}\right] 19-$-nor-DOC contained carbon-14. Presently, our evidence for the identity of the latter metabolites is presumptive, based on the polarities of these metabolites relative to those of the known metabolites of DOC. From these findings, we conclude that plasma DOC is not converted to 19-nor-DOC.

The production rate of 19-nor-DOC in one of the men of this study, computed from the specific activity of isolated urinary $\left[{ }^{3} \mathrm{H}\right] 19-$-nor-DOC (glucuronoside) and the amount of injected tracer, was $\cong 16 \mu \mathrm{g} / \mathrm{d}$ and in both of the young women of the study, it was $\cong 10 \mu \mathrm{g} / \mathrm{d}$. Thus, dilution of the injected $\left[{ }^{3} \mathrm{H}\right] 19-$ nor-DOC occurs in vivo. This was demonstrated by measurement of mass and radioactivity in purified $\left[{ }^{3} \mathrm{H}\right] 19$ -
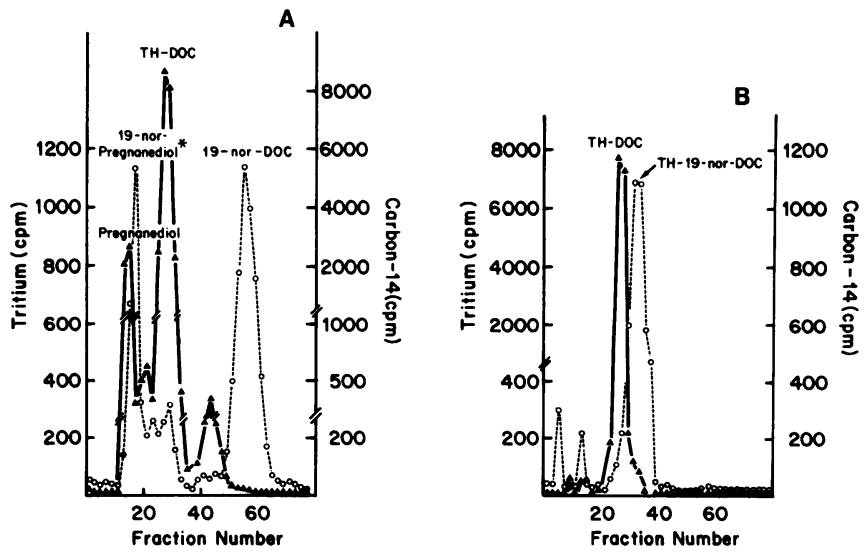

Figure 2. Chromatogram after liquid-liquid partition column chromatography of fractions 78-86 $(A)$ of the gradient-elution column chromatogram as shown in Fig. 1 and fractions 89-98 $(B)$ of the gradient-elution column chromatogram as shown in Fig. 1. The asterisks are indicative of tentative identification by isopolarity in multiple chromatographic systems. --- O---, tritium; - - , carbon-14. 
nor-DOC isolated from urine after hydrolysis with $\beta$-glucuronidase. Thus, the 19-nor-DOC that is produced in men and women is not that which is excreted in urine in nonconjugated form. We cannot conclude that the 19-nor-DOC produced in vivo enters blood but we can conclude that a portion thereof suffers a metabolic fate similar to that of infused $\left[{ }^{3} \mathrm{H}\right] 19$-norDOC, i.e., excretion as 19-nor-DOC-glucuronoside.

\section{Discussion}

The potent mineralocorticosteroid, 19-nor-DOC, may serve a role in the pathogenesis of certain forms of hypertension, especially in experimental animals $(4,5,7)$. Yet, the origin of the 19-nor-DOC that is found in urine of rats and humans $(5$, $7-10,24,25)$ is unknown. Intuitively, one would expect that such a steroid would be secreted by the adrenal cortex or else would be formed in extraadrenal tissues from DOC. It has not been possible, however, to demonstrate the biosynthesis of 19 nor-DOC in adrenal tissue $(11,12)$.

Heretofore, investigators have quantified the amount of 19-nor-DOC that was present as the free steroid in urine of experimental animals $(5,7,24)$ and humans $(8-10,24,25)$. In the present investigation, however, we found that little of intravenously infused radiolabeled 19-nor-DOC was excreted as a free steroid. Indeed, only $1 \%$ or less of the radioactivity of injected $\left[{ }^{3} \mathrm{H}\right] 19-$-nor-DOC could be extracted from urine before hydrolysis of steroid conjugates, and of this amount of radioactivity, none was attributable to $\left[{ }^{3} \mathrm{H}\right] 19$-nor-DOC. Rather, the vast majority of the radioactivity in urine was recovered by extraction after treatment of urine with $\beta$-glucuronidase. Nonetheless, there was immunoreactive free 19-nor-DOC in the urine of each test subject. Based on these findings, we suggest that most of the metabolites of circulating (or infused) 19-nor-DOC are excreted after conjugation with glucuronic acid, and, in the case of 19-nor-DOC per se, such a putative conjugate must be the 21 -glucuronoside. By way of contrast, none of the intravenously administered $\left[{ }^{14} \mathrm{C}\right] \mathrm{DOC}$ was excreted as $\left[{ }^{14} \mathrm{C}\right] \mathrm{DOC}$-glucuronoside.

From these several observations, we conclude that free 19nor-DOC in urine is formed in kidney from a precursor other than DOC or else is formed in kidney or in urine nonenzymatically from a precursor such as the 19-carboxylic acid derivative of DOC, a compound that is known to be synthesized in rat adrenal tissue in vitro (12) and, possibly, in man. This obtains since if the 19-nor-DOC that is found in urine as the free steroid were formed in tissues other than kidney, the likely metabolic fate of such a steroid would be similar to that of intravenously infused $\left[{ }^{3} \mathrm{H}\right] 19$-nor-DOC; if not, there seems no reasonable manner by which it could reach urine as free 19-nor-DOC. On the other hand, it also is apparent that there is, in vivo, production of 19-nor-DOC that is independent of that which is formed in kidney or urine and excreted as the free compound. This obtains since the $\left[{ }^{3} \mathrm{H}\right] 19$-nor-DOC (glucuronoside) was diluted by nonradiolabeled 19-nor-DOC as evidenced by the specific activity of urinary $\left[{ }^{3} \mathrm{H}\right] 19$-nor-DOC (glucuronoside). From the specific activity of this metabolite and the amount of $\left[{ }^{3} \mathrm{H}\right] 19$-nor-DOC infused, the production rate of 19-nor-DOC was computed to be $\cong 10-16 \mu \mathrm{g} / \mathrm{d}$ ( $n$ $=3$ ).

Steroid 21-hydroxylase activity has been demonstrated in a number of extraglandular tissues in humans (cf. 26) and the 21-hydroxylation of plasma progesterone is known to account, in large measure, for the striking increase in the rate of production of DOC in women during the midluteal phase of the ovarian cycle $(14,27)$ and during pregnancy $(13,15)$. Nonetheless, as shown in the present study, plasma DOC, in men and women, is not metabolized by way of 19-nor-DOC, nor by way of excretion into urine as nonconjugated DOC.

In the human, the metabolism of $\mathrm{DOC}$ and related compounds is unique. $\mathrm{DOC}$ is not converted to plasma $\mathrm{DOC}-\mathrm{SO}_{4}$ and $\mathrm{DOC}-\mathrm{SO}_{4}$ is not metabolized by way of DOC (28), except in placenta (29) and by bacterial enzymes in intestine (28, 30, 31 ). On the other hand, DOC is converted to $\mathrm{DOC}-\mathrm{SO}_{4}$ in liver; the $\mathrm{DOC}^{-\mathrm{SO}_{4}}$ formed in liver, however, does not enter blood, but rather enters bile quantitatively (28). The metabolic clearance rate of this steroid sulfoconjugate is one or two orders of magnitude greater than the clearance of any other steroid sulfate known (32), presumably because of the unique biliary metabolic disposition of $\mathrm{DOC}^{-\mathrm{SO}_{4}}$. Thus, we find that DOC-SO $\mathrm{S}_{4}$, and now, the potent mineralocorticosteroid 19nor-DOC are not formed from plasma DOC. The origin of these compounds is undefined.

These several findings, taken together, are supportive of the conclusion that the biosynthesis and metabolism of DOC and related substances are not typical of the metabolic fate that we have come to recognize as being true for other $C_{21}$ steroids. In addition, extraadrenal steroid 21-hydroxylase activity appears to be regulated by environmental factors since estrogen acts to increase the activity of this enzyme in extraadrenal tissues (33), and in spleen, extraadrenal steroid 21hydroxylase activity is altered by immunologic challenges (34). In consideration of the biological potency of several of the compounds involved, it seems reasonable to suspect that aberrations in one or more of these unusual metabolic processes could give rise to abnormalities that involve mineralocorticosteroid action. In this context, it is important to note that Shackleton (35) could find little or no tetrahydro-19-nor-DOC in urine of persons with $17 \alpha$-hydroxylase deficiency. This finding, as he properly notes, also stands in evidence against the proposition that 19-nor-DOC is synthesized in the adrenal. Moreover, our findings also are supportive of the proposition that free urinary 19-nor-DOC is not formed from plasma DOC. Based on these findings, we postulate that the free 19nor-DOC in urine is formed either in kidney or else nonenzymatically in kidney or in urine from 19-oic-DOC that can be synthesized in adrenal tissue (12). In support of the latter possibility are the recent findings of Griffing et al. (36) that the levels of 19-nor-DOC in plasma and urine of a woman with $17 \alpha$-hydroxylase deficiency are increased as are the plasma levels of a precursor (possibly 19-oic-DOC) that is converted to 19-nor-DOC by acidification of plasma. These investigators demonstrated that plasma levels of 19-nor-DOC and the precursor are decreased by treatment with dexamethasone and increased by treatment with ACTH (36). In addition, Griffing et al. (37) obtained evidence that 19-nor-DOC production is not regulated by the renin-angiotensin system. Thus, there is considerable evidence in favor of the proposition that 19-norDOC in urine arises in kidney or in urine from a precursor that is secreted by the adrenal.

\section{Acknowledgments}

We thank Frank Hereford, Steve Robinson, and Grace Lau for skilled technical assistance and Rosemary Bell for expert editorial assistance.

This investigation was supported, in part, by U. S. Public Health Service grants nos. 5-P50-HD11149 and 2-P01-AG00306. Dr. Guerami was a postdoctoral trainee supported, in part, by U. S. Public Health Service Training grant no. 1-T32-HD07190. 


\section{References}

1. Axelrod, B. J., J. E. Cates, B. B. Johnson, and J. A. Luetscher, Jr. 1954. Bioassay of mineralocorticoids: relationship of structure to physiological activity. Endocrinology. 55:568-574.

2. Kagawa, C. M., and C. G. Van Arman. 1957. Sodium retaining activity of 19-nor-steroids in adrenalectomized rats. Proc. Soc. Exp. Biol. Med. 94:444-447.

3. Funder, J. W., J. Mercer, B. Ingram, D. Feldman, K. Wynne, and W. R. Adam. 1978. 19-Nor-deoxycorticosterone (19-nor-DOC): mineralocorticoid receptor affinity higher than aldosterone, electrolyte activity lower. Endocrinology. 103:1514-1517.

4. Hall, C. E., C. E. Gomez-Sanchez, O. B. Holland, and D. Nasseth. 1979. Influence of 19-nor-deoxycorticosterone on blood pressure, saline consumption and serum electrolytes, corticosterone and renin activity. Endocrinology. 105:600-604.

5. Gomez-Sanchez, C. E., O. B. Holland, B. A. Murray, H. A. Lloyd, and L. Milewich. 1979. 19-Nor-deoxycorticosterone: a potent mineralocorticoid isolated from the urine of rats with regenerating adrenals. Endocrinology. 105:708-711.

6. Wynne, K. N., J. Mercer, J. R. Stockigt, and J. W. Funder. 1980. 19-Nor-analogs of adrenal steroids: mineralocorticoid and glucocorticoid receptor activity. Endocrinology. 107:1278-1280.

7. Dale, S. L., M. M. Holbrook, P. Komanicky, and J. C. Melby 1982. Urinary 19-nor-deoxycorticosterone excretion in the spontaneously hypertensive rats. Endocrinology. 110:1989-1993.

8. Dale, S. L., M. M. Holbrook, and J. C. Melby. 1981. 19-Nordeoxycorticosterone in the neutral fraction of human urine. Steroids. 37:103-109.

9. Griffing, G. T., S. L. Dale, M. M. Holbrook, and J. C. Melby. 1983. The regulation of urinary free 19-nor-deoxycorticosterone and its relation to systemic arterial blood pressure in normotensive and hypertensive subjects. J. Clin. Endocrinol. Metab. 56:99-103.

10. Griffing, G. T., S. L. Dale, M. M. Holbrook, and J. C. Melby. 1981. 19-Nor-deoxycorticosterone excretion in healthy and hypertensive subjects. Trans. Assoc. Am. Physicians. 94:301-309.

11. Dale, S. L., M. M. Holbrook, and J. C. Melby. 1980. Identification of 19-hydroxy-deoxycorticosterone in regenerating rat adrenal incubations. Steroids. 36:601-609.

12. Gomez-Sanchez, C. E., E. P. Gomez-Sanchez, C. H. L. Shackleton, and L. Milewich. 1982. Identification of 19-hydroxydeoxycorticosterone, 19-oxo-deoxycorticosterone, and 19-oic-deoxycorticosterone as products of deoxycorticosterone metabolism by rat adrenals. Endocrinology. 110:384-389.

13. Winkel, C. A., L. Milewich, C. R. Parker, Jr., N. F. Gant E. R. Simpson, and P. C. MacDonald. 1980. Conversion of plasma progesterone to deoxycorticosterone in men, nonpregnant and pregnant women, and adrenalectomized subjects: evidence for steroid 21-hydroxylase activity in nonadrenal tissues. J. Clin. Invest. 66:803-812.

14. Winkel, C. A., C. R. Parker, Jr., E. R. Simpson, and P. C. MacDonald. 1980. Production rate of deoxycorticosterone in women during the follicular and luteal phases of the ovarian cycle: the role of extraadrenal 21-hydroxylation of circulating progesterone in deoxycorticosterone production. J. Clin. Endocrinol. Metab. 51:1354-1358.

15. Casey, M. L., A. Guerami, C. A. Winkel, and P. C. MacDonald. 1984. The origin and metabolic fate of deoxycorticosterone and deoxycorticosterone sulfate in pregnant women and their fetuses. $J$. Steroid Biochem. 20:237-243.

16. Winkel, C. A., E. R. Simpson, L. Milewich, and P. C. MacDonald. 1980. Deoxycorticosterone biosynthesis in human kidney: potential for formation of a potent mineralocorticosteroid in its site of action. Proc. Natl. Acad. Sci. USA. 77:7069-7073.

17. Winkel, C. A., M. L. Casey, E. R. Simpson, and P. C. MacDonald. 1981. Deoxycorticosterone biosynthesis from progesterone in kidney tissue of the human fetus. J. Clin. Endocrinol. Metab. 53: 10-15.

18. Casey, M. L., and P. C. MacDonald. 1982. Formation of deoxycorticosterone from progesterone in extraadrenal tissues: demonstration of steroid 21-hydroxylase activity in human aorta. J. Clin. Endocrinol. Metab. 55:804-806.
19. Casey, M. L., C. A. Winkel, and P. C. MacDonald. 1983. Conversion of progesterone to deoxycorticosterone in the human fetus: steroid 21-hydroxylase activity in fetal tissues. J. Steroid Biochem. 18: 449-452.

20. Gomez-Sanchez, C. E., E. P. Gomez-Sanchez, and R. J. Upcavage. 1983. Radioimmunoassay for urinary free 19-nor-deoxycorticosterone: effects of adrenocorticotropin and sodium depletion. Endocrinology. 112:2046-2049.

21. Burstein, S., and S. Lieberman. 1958. Hydrolysis of keto-steroid hydrogen sulfates by solvolysis procedure. J. Biol. Chem. 233:331335.

22. Siiteri, P. K. 1963. The isolation of urinary estrogens and determination of their specific activities following the administration of radioactive precursors to humans. Steroids. 2:687-712.

23. Zaffaroni, A., H. J. Ringold, G. Rosenkranz, F. Sondheimer, G. H. Thomas, and C. Djerassi. 1958. Steroids. C. Synthesis of 19nor- $\Delta^{4}$-pregnene-11 $\beta, 17 \alpha, 21$-triol-3,20-dione (19-nor-hydrocortisone) and related 19-nor-adrenal hormones. J. Am. Chem. Soc. 80:61106114.

24. Melby, J. C., S. L. Dale, M. M. Holbrook, and G. T. Griffing. 1982. 19-Nor-corticoids in experimental and human hypertension. Clin. Exper. Hypertens. Part A Theory Pract. 4:1851-1867.

25. Griffing, G. T., S. L. Dale, M. M. Holbrook, and J. C. Melby. 1983. 19-Nor-deoxycorticosterone excretion in primary aldosteronism and low renin hypertension. J. Clin. Endocrinol. Metab. 56:218-221.

26. Casey, M. L., and P. C. MacDonald. 1982. Extraadrenal formation of a mineralocorticosteroid: deoxycorticosterone and deoxycorticosterone sulfate biosynthesis and metabolism. Endocrine Rev. 3: 396-403.

27. Parker, C. R., Jr., C. A. Winkel, A. J. Rush, Jr., J. C. Porter, and P. C. MacDonald. 1981. Plasma concentrations of 11-deoxycorticosterone in women during the menstrual cycle. Obstet. Gynecol. 58: 26-30.

28. Casey, M. L., and P. C. MacDonald. 1982. Metabolism of deoxycorticosterone and deoxycorticosterone sulfate in men and women. J. Clin. Invest. 70:312-319.

29. Mathis, J. M., J. D. Johnston, P. C. MacDonald, and M. L. Casey. 1983. Steroid 21-sulfatase activity in human placenta. J. Steroid Biochem. 18:575-579.

30. Bokkenheuser, V. D., J. Winter, P. Dehaeya, and W. G. Kelly. 1977. Isolation and characterization of human fecal bacteria capable of 21-dehydroxylating corticoids. Appl. Environ. Microbiol. 34:571575 .

31. Winter, J., and V. D. Bokkenheuser. 1978. 21-Dehydroxylation of corticoids by anaerobic bacteria isolated from human fecal flora. $J$. Steroid Biochem. 9:379-384.

32. Guerami, A., M. L. Casey, and P. C. MacDonald. 1983. Metabolic clearance rate of deoxycorticosterone sulfate in men and women. J. Steroid Biochem. 19:1173-1177.

33. MacDonald, P. C., S. Cutrer, S. C. MacDonald, M. L. Casey, and C. R. Parker, Jr. 1982. Regulation of extraadrenal steroid 21hydroxylase activity: increased conversion of plasma progesterone to deoxycorticosterone during estrogen treatment of women pregnant with a dead fetus. J. Clin. Invest. 69:469-478.

34. Winkel, C. A., and D. L. Danley. 1983. The effect of immunologic challenge on splenic steroid 21-hydroxylase activity in the guinea pig. Proc. 65th Meeting Endocrine Soc., San Antonio, TX. 329. (Abstr.)

35. Shackleton, C. H. L. 1982. Evidence against significant 19-nordeoxycorticosterone production in patients with $17 \alpha$-hydroxylase deficiency. Clin. Exper. Hypertens. Part A Theory Pract. 4:1529-1539.

36. Griffing, G. T., T. E. Wilson, M. M. Holbrook, S. L. Dale, T. K. Jackson, I. Ullrich, and J. C. Melby. 1984. Plasma and urinary 19-nor-deoxycorticosterone in $17 \alpha$-hydroxylase deficiency syndrome. J. Clin. Endocrinol. Metab. 59:1011-1015.

37. Griffing, G. T., T. E. Wilson, and J. C. Melby. 1984. Transitory increment in urinary free 19-nor-deoxycorticosterone after diureticinduced renin stimulation. J. Clin. Endocrinol. Metab. 59:931-935. 